\title{
Immunostimulatory Tim-1-specific antibody deprograms Tregs and prevents transplant tolerance in mice
}

\author{
Nicolas Degauque, ${ }^{1}$ Christophe Mariat, ${ }^{1}$ James Kenny,, Dong Zhang, ${ }^{1}$ Wenda Gao, ${ }^{1}$ \\ Minh Diem Vu, ${ }^{1}$ Sophoclis Alexopoulos, ${ }^{1}$ Mohammed Oukka, ${ }^{2}$ Dale T. Umetsu, ${ }^{3}$ \\ Rosemarie H. DeKruyff, ${ }^{3}$ Vijay Kuchroo, ${ }^{2}$ Xin Xiao Zheng, ${ }^{1}$ and Terry B. Strom ${ }^{1}$
}

${ }^{1}$ Division of Transplant Immunology and Transplant Research Center, Beth Israel Deaconess Medical Center, Harvard Medical School, Boston, Massachusetts, USA. ${ }^{2}$ Center for Neurological Diseases, Brigham and Women's Hospital, Harvard Medical School, Boston, Massachusetts, USA. ${ }^{3}$ Division of Immunology, Children's Hospital Boston, and Harvard Medical School, Boston, Massachusetts, USA.

\begin{abstract}
$\mathrm{T}$ cell Ig mucin (Tim) molecules modulate $\mathrm{CD}^{+} \mathrm{T}$ cell responses. In keeping with the view that Tim-1 generates a stimulatory signal for $\mathrm{CD}^{+} \mathrm{T}$ cell activation, we hypothesized that an agonist $\mathrm{Tim}-1$-specific $\mathrm{mAb}$ would intensify the $\mathrm{CD}^{+} \mathrm{T}$ cell-dependant allograft response. Unexpectedly, we determined that a particular Tim-1-specific mAb exerted reciprocal effects upon the commitment of alloactivated $T$ cells to regulatory and effector phenotypes. Commitment to the Th1 and Th17 phenotypes was fostered, whereas commitment to the Treg phenotype was hindered. Moreover, ligation of Tim-1 in vitro effectively deprogrammed Tregs and thus produced Tregs unable to control $\mathrm{T}$ cell responses. Overall, the effects of the agonist Tim-1-specific mAb on the allograft response stemmed from enhanced expansion and survival of $\mathrm{T}$ effector cells; a capacity to deprogram natural Tregs; and inhibition of the conversion of naive $\mathrm{CD} 4^{+} \mathrm{T}$ cells into Tregs. The reciprocal effects of agonist Tim-1-specific mAbs upon effector $T$ cells and Tregs serve to prevent allogeneic transplant tolerance.
\end{abstract}

\section{Introduction}

$\mathrm{T}$ cell Ig mucin (Tim) molecules are structurally related to type I membrane glycoproteins expressed on T cells (1). Polymorphisms within the Tim gene locus are associated with susceptibility to atopy and autoimmunity, which suggests that Tim proteins modulate $\mathrm{CD} 4^{+} \mathrm{T}$ cell responses (2-4). Indeed, recent reports have confirmed the role of Tim molecules in regulating the expansion and effector function of Th1 and Th2 cells (5-8). For example, Tim-3 and Tim- 2 negatively regulate $\mathrm{Th} 1$ and Th 2 responses, respectively $(9,10)$, through the delivery of inhibitory or death signals into select $\mathrm{CD} 4^{+} \mathrm{T}$ cell populations.

Based on genetic linkage and epidemiologic studies, it was reasonable to assume that ligation of Tim- 1 might preferentially skew the immune response toward a Th2 phenotype $(11,12)$, because anti-Tim-1 amplifies Th2-type cytokine production in a Th2biased experimental model of airway hyperreactivity (13). Unlike ligation of Tim-3 or Tim-2, Tim-1 ligation heightens T cell activation (13). Naive $\mathrm{CD}^{+}+\mathrm{T}$ cells upregulate Tim- 1 expression early after activation, and Tim-1 cell-surface expression is maintained through differentiation into the Th1 or Th2 phenotype $(13,14)$. Tim-4, a molecule expressed by DCs (15), is a ligand of Tim-1 (15). Cross-linking of Tim- 1 on the surface of $\mathrm{T}$ cells in vitro by Tim- 4 Ig enhances T cell proliferation and production of Th1 and Th2 cytokines. In vivo administration of Tim-4 Ig during an ongoing immune response creates similar effects (15).

In keeping with the view that Tim-1 generates a stimulatory signal for T cell activation (16), we demonstrate that an agonist anti-

Nonstandard abbreviations used: CTLA-4, CTL-associated antigen-4; GITR, glucocorticoid-induced TNF receptor family-related protein; MLR, mixed lymphocyte reaction; Teff, effector T cell; TIM, T cell Ig mucin.

Conflict of interest: The authors have declared that no conflict of interest exists. Citation for this article: J. Clin. Invest. 118:735-741 (2008). doi:10.1172/JCI32562.
Tim-1 mAb $(13,17)$ intensifies the allograft response and prevents development of $\mathrm{T}$ cell tolerance. Unexpectedly, we determined that agonist anti-Tim-1 mAb exerts reciprocal effects upon the commitment of alloactivated $\mathrm{T}$ cells to regulatory and effector phenotypes. In the context of alloimmunity, we demonstrate that Tim-1 greatly enhances proinflammatory (Th1 and Th17) cell-mediated responses and hampers the development of peripheral tolerance. In addition, we now report on the capacity of Tim-1 to deprogram the $\mathrm{CD} 4^{+} \mathrm{Foxp}^{+} \mathrm{T}$ cell-dependent regulatory loops and to promote differentiation of Th17 cells. Collectively, our data indicate that ligation of Tim-1 reciprocally alters commitment of alloreactive $\mathrm{CD} 4^{+} \mathrm{T}$ cells to the $\mathrm{CD} 4^{+} \mathrm{Foxp} 3^{+}$and $\mathrm{CD} 4^{+} \mathrm{Th} 17^{+}$phenotypes.

\section{Results}

3B3 anti-Tim-1 $m A$ Ab strengthens Th1/Th17 polarization and enhances the expansion and survival of $C D 4^{+}$and $C D 8^{+}$alloreactive cells in vitro. Umetsu et al. developed the 3B3 agonist type anti-Tim-1 mAb and provided the first mechanistic insights into the function of Tim-1 (13). Application of 3B3 anti-Tim-1 mAb heightens T cell activation and prevents the development of respiratory tract tolerance in a Th2-driven model of asthma (13). We have now used 3B3 anti-Tim- $1 \mathrm{mAb}$ to study the role of Tim-1 in the in vivo allograft response, a prototypic Th1 effector $\mathrm{T}$ cell-driven (Teff-driven) process, and in vitro, e.g., the mixed lymphocyte reaction (MLR) (Figure 1). Mature allogeneic DCs (i.e., DBA/2 bone marrowderived $\mathrm{CD} 80^{+} \mathrm{CD} 40^{+} \mathrm{DCs}$ ) were used to stimulate CFSE-labeled $\mathrm{C} 57 \mathrm{BL} / 6 \mathrm{CD} 4^{+} \mathrm{CD} 25^{-}$and $\mathrm{CD} 8^{+} \mathrm{CD} 25^{-} \mathrm{T}$ cells in the presence of $3 \mathrm{~B} 3$ anti-Tim-1 mAb or an isotype control antibody. The proliferative response of CFSE-stained alloreactive $T$ cells was analyzed by flow cytometry (18) (Figure 1A). The proliferative response of $\mathrm{CD}^{+} \mathrm{CD} 25^{-}$and $\mathrm{CD} 8^{+} \mathrm{CD} 25^{-} \mathrm{T}$ cells in the MLR was accelerated and exaggerated with provision of $3 \mathrm{~B} 3$ anti-Tim- $1 \mathrm{mAb}$ (Figure $1, \mathrm{~A}$ and $\mathrm{B}$ ). In cultures containing anti-Tim- $1 \mathrm{mAb}, 3-4$ discrete 
A

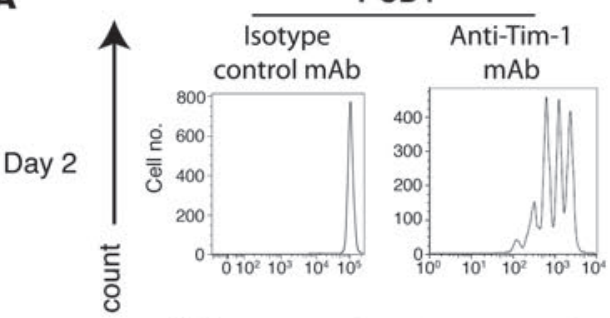

T CD4

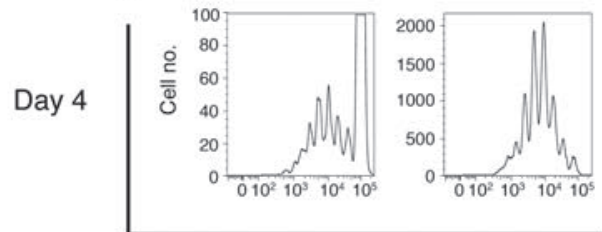

B

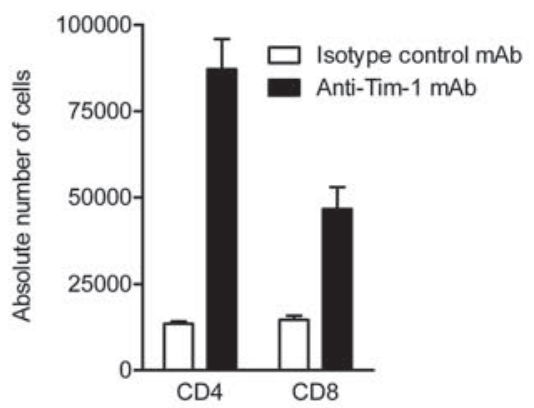

D
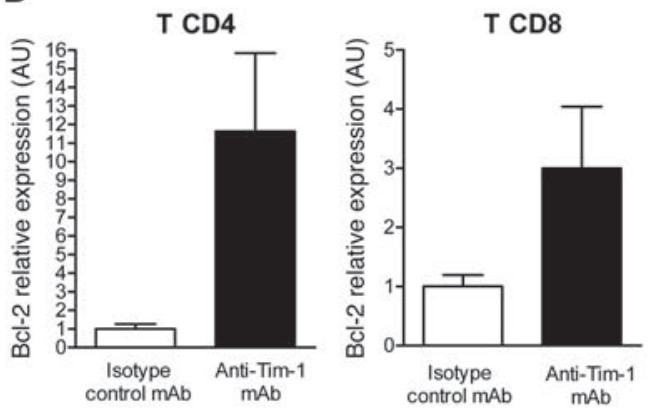
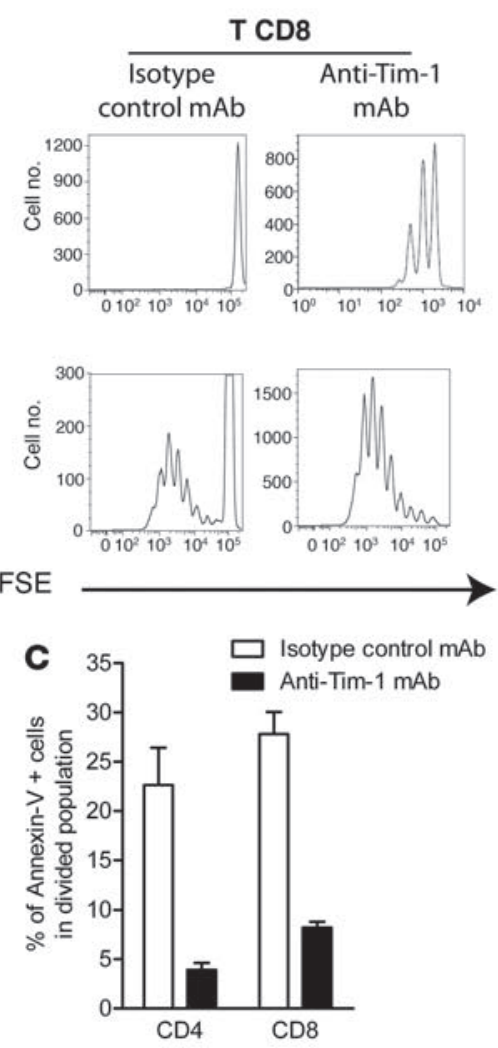

E
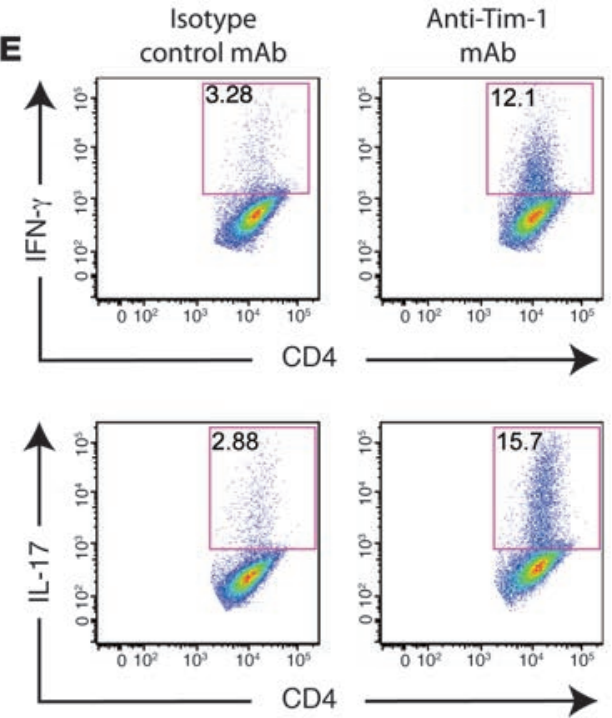

Figure 1

Effect of anti-Tim-1 mAb on alloreactive $\mathrm{CD}^{+}{ }^{+}$and $\mathrm{CD} 8^{+}$Teff responses in vitro. CFSE-labeled $\mathrm{C} 57 \mathrm{BL} / 6 \mathrm{CD}^{+}{ }^{\mathrm{CD}} 25^{-}$and CD8 ${ }^{+}$CD25- cells were stimulated with highly mature allogeneic $\mathrm{DBA} / 2 \mathrm{DCs}$ in the presence of anti-Tim-1 mAb or IgG2a isotype control mAb. Analysis of the CFSE profile (A) and enumeration of the absolute number of $\mathrm{CD}_{3}{ }^{+} \mathrm{CD} 4^{+}$or $\mathrm{CD} 8^{+}$cells (B) were used to assess the proliferation and expansion of alloreactive T cells after 2 (A) and 4 (A and $\mathbf{B}$ ) days of culture. (C) The effect of anti-Tim-1 mAb on the proportion of cells programmed for apoptosis (annexin $\mathrm{V}^{+}$cells) in the proliferating cell population was assessed after 4 days of culture. (D) To gain further insight, we analyzed $\mathrm{Bcl}-2$ transcript expression in alloreactive $\mathrm{CD} 4^{+}$or $\mathrm{CD} 8^{+}$cells after 2 days of culture. (E) Finally, secretion of IFN- $\gamma$ (Th1) and IL-17 (Th17) by alloreactive Teffs after 4 days of culture was assessed by intracellular staining. Data represent 1 of 8 independent experiments $(\mathbf{A}$ and $\mathbf{E})$ or are presented as the mean \pm SEM of at least 5 independent experiments (B-D). generations of $\mathrm{T}$ cell proliferation were detectable as early as day 2 of culture, while proliferation was not yet evident at day 2 in control MLR cultures (Figure 1A). Furthermore, by day 4, the addition of $3 \mathrm{~B} 3$ anti-Tim- $1 \mathrm{mAb}$ to the MLR resulted in a substantial increase in $\mathrm{CD}^{+}$and $\mathrm{CD}^{+} \mathrm{T}$ cell accumulation (6.5- and 3.2-fold respectively) as compared with isotype control MLRs (Figure 1B). Detailed analysis of the CFSE profile strongly indicated that the scope of $\mathrm{T}$ cell proliferation induced by agonist anti-Tim-1 mAb cannot be restricted to the alloreactive subpopulation (Figure 1A). The proportion of proliferating $\mathrm{T}$ cells exceeds any estimation of the frequency of alloreactive $T$ cells. Hence, we tested whether agonist anti-Tim-1 mAb elicited $\mathrm{T}$ cell proliferation when syngeneic, not allogeneic, mature DCs are used (Supplemental Figure 1; supplemental material available online with this article; doi:10.1172/ JCI32562DS1). Surprisingly, anti-Tim-1 mAb elicits robust $\mathrm{T}$ cell proliferation in the presence of syngeneic DCs. These data indicate that in the presence of syngeneic DCs, 3B3 anti-Tim- $1 \mathrm{mAb}$ acts a polyclonal stimulant.

In order to determine whether anti-Tim-1 mAb might promote the survival of $\mathrm{T}$ cells responding in the MLR, we assessed the proportion of cultured $\mathrm{T}$ cells programmed for apoptosis by counterstaining CFSE-labeled $\mathrm{CD}^{+}$and $\mathrm{CD}^{+}$cells with annexin $\mathrm{V}$ (Figure 1C). As we have previously reported (19), the intensity of annexin $V$ staining gradually increases with each cell division, reflecting the 

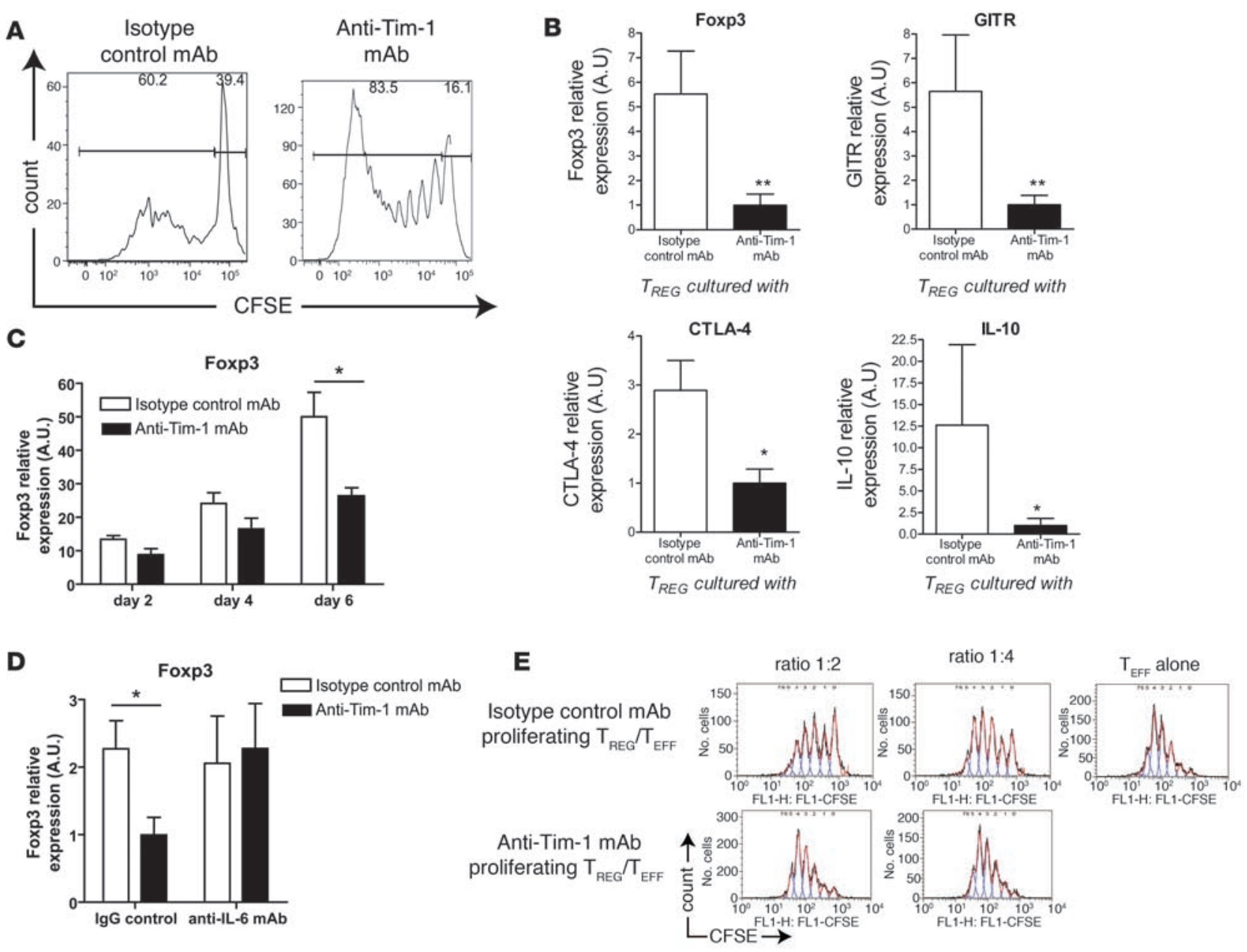

Figure 2

Effect of 3B3 anti-Tim-1 mAb on alloreactive Tregs in vitro. (A) CFSE-labeled CD4+CD25+ Tregs were stimulated with mature allogeneic DCs in the presence of anti-Tim-1 mAb or IgG2a isotype control mAb. Proliferation of alloreactive CD4+CD25+ Tregs was assessed by flow cytometry after 6 days of culture through analysis of the CFSE profile of CD4 ${ }^{+} T$ cells. (B) Next, proliferating CFSEdim $C D 4^{+} C D 25^{+}$Tregs were isolated by flow cytometry for RNA extraction, and relative expression of Treg-associated transcripts was determined by quantitative real-time PCR. (C) FACS-sorted GFP(Foxp3)+ Tregs from Foxp3-GFP knock-in mice were cocultured with mature allogeneic DCs in the presence of anti-Tim-1 mAb or lgG2a isotype control mAb. Foxp3 gene expression was assessed by quantitative real-time PCR on days 2, 4 and 6. (D) Neutralizing anti-IL-6 mAb or isotype control mAb was added to the culture of GFP(Foxp3)+ Tregs with mature allogeneic DCs. After 6 days of culture, Foxp3 expression was analyzed by quantitative real-time PCR. (E) CFSE-labeled CD45.1+ Teffs (CD4+CD25-) were stimulated by plate-bound anti-CD3 and soluble anti-CD28 mAb for 4 days (Teff alone), or cocultured with alloreactive CD45.2+ Tregs, previously stimulated either in the presence of IgG2a isotype control mAb (Isotype control mAb proliferating Treg/Teff) or in the presence of anti-Tim-1 mAb (Anti-Tim $1 \mathrm{mAb}$ proliferating Treg/Teff). Proliferation of Teffs was assessed by flow cytometry, based on the CFSE profile of CD45.1 ${ }^{+}$cells. Data are representative of 4 (A and $\mathbf{E}$ ) independent experiments or are presented as the mean \pm SEM of 4 different independent experiments (B-D).

physiological process of activation-induced apoptotic cell death. Interestingly, the proportion of proliferating annexin $\mathrm{V}^{+} \mathrm{CD} 4^{+}$and $\mathrm{CD}^{+} \mathrm{T}$ cells was markedly reduced in anti-Tim- $1 \mathrm{mAb}-$ supplemented MLRs as compared with isotype control-treated cultures (Figure 1C). Hence, expansion of T cells induced by $3 \mathrm{~B} 3$ anti-Tim-1 $\mathrm{mAb}$ in the MLR is due to accelerated and exaggerated cell division as well as a survival advantage directly conferred by Tim-1-dependent signals. In keeping with these observations, amplified expression of the antiapoptotic Bcl-2 gene (20) was detected in MLRactivated, proliferating $\mathrm{CD} 4^{+}$and $\mathrm{CD} 8^{+} \mathrm{T}$ cells harvested from the anti-Tim-1 mAb-treated cultures (Figure 1D).

Next, we sought to characterize whether supplementation of the MLR with anti-Tim-1 mAb altered the balance of Th1/Th2/
Th17 cells. Provision of agonist anti-Tim-1 mAb to the culture enhanced the commitment of proinflammatory Th1 and Th17 T cells, as assessed by intracellular immunostaining for IFN- $\gamma$ and IL-17 (Figure 1E). These profiles were further confirmed using quantitative real-time PCR (Supplemental Figure 2). In contrast, gene expression for IL-4, the prototypic Th2-type cytokine, was downregulated in cultures supplemented with agonist anti-Tim-1 $\mathrm{mAb}$ (Supplemental Figure 2). Thus, activation of the Tim-1 pathway promotes the commitment of naive $\mathrm{T}$ cells toward a Th1/ Th17-biased proinflammatory-type response.

3B3 anti-Tim-1 mAb dampens expression of regulatory-associated genes and the ability of alloreactive Tregs to suppress Teff responses in vitro. A heightened Th1-type alloreactive response can result from 
A

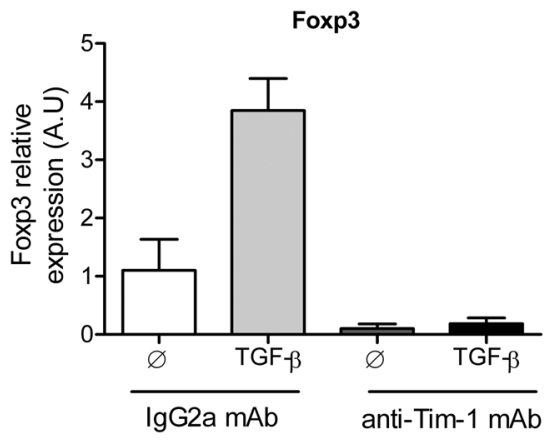

B
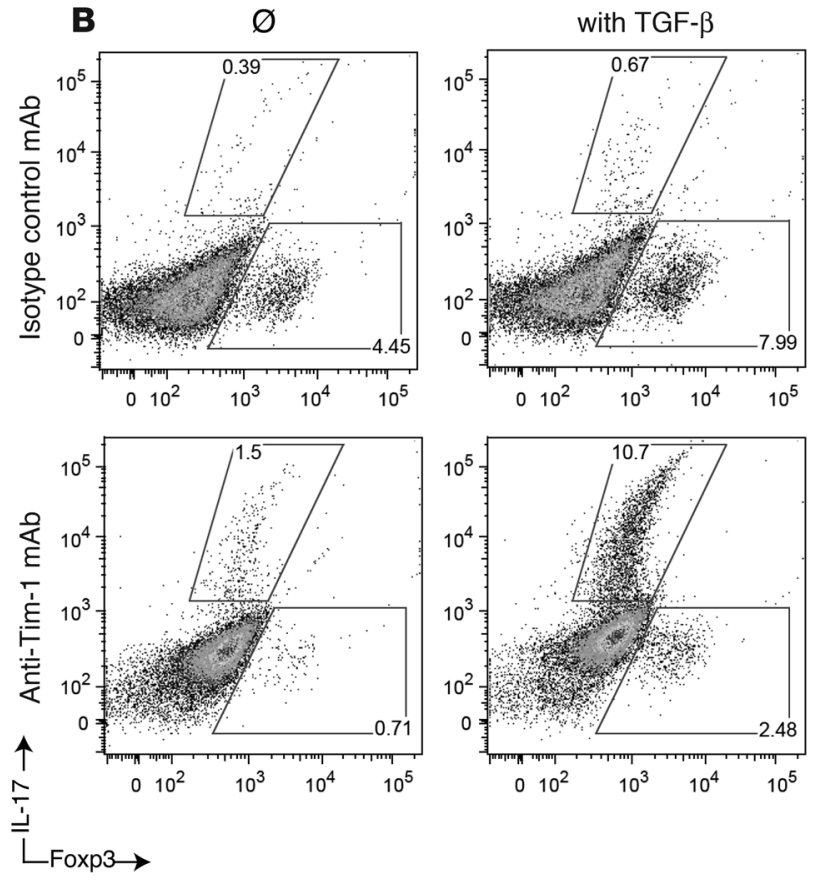

weakened Treg-dependent $\mathrm{T}$ cell immunoregulation. Moreover, a reciprocal relationship between commitment to the Th17 and Treg phenotype has been noted in some settings (21). As 3B3 antiTim-1 mAb heightens Th1/Th17 type alloactivation, we sought to determine whether TIM-1-generated signals may impair Tregdependent immunoregulation.

Interaction of Tregs with highly mature syngeneic DCs rescues Tregs from anergy in vitro (22). We and others have extended this observation to the alloimmune response and have consistently observed substantial proliferation of TCR-transgenic (23) or polyclonal (24) Tregs when MLRs utilize mature allogeneic DCs as stimulator cells. Using this system, we have examined effect of anti-Tim-1 mAb upon the in vitro expansion of alloreactive Tregs (Figure 2).

Proliferation of $\mathrm{CD} 4{ }^{+} \mathrm{CD} 25^{+} \mathrm{C} 57 \mathrm{BL} / 6$ Tregs stimulated with mature DBA/2 DCs was markedly enhanced by provision of 3B3 anti-Tim-1 $\mathrm{mAb}$ (Figure 2A), suggesting at first that anti-Tim-1 $\mathrm{mAb}$ stimulation might amplify the proliferative response of activated Tregs. Thus, we next analyzed transcription of Treg-associated genes in both anti-Tim-1 mAb-treated and control MLRs, focusing specifically on the proliferating (CFSE ${ }^{10}$ ) Treg population. We observed that expression of Foxp3 transcription factor, the Treg master switch (25), was downregulated in the presence

\section{Figure 3}

3B3 anti-Tim-1 mAb prevents the de novo induction of Foxp3+ Tregs from Teffs and promotes the differentiation of Th17 cells. CD4+GFP(Foxp3)- T cells isolated from naive C57BL/6 Foxp3-GFP knock-in mice were cultured with highly mature syngeneic $D C s$ plus anti-CD3 mAb in the presence of IgG2a mAb or anti-Tim-1 mAb and in the presence or absence of TGF- $\beta$. Induction of Foxp3 expression was assessed 3 days later by quantitative real-time PCR (A) or by intracellular staining of Foxp3 (B). Quantification of conversion of CD4+GFP(Foxp3)- to CD4+Foxp3+ Tregs at the level of protein expression was determined by gating onto the CD4+ fraction. Differentiation of Th17 cells was assessed by intracellular staining of IL-17 and quantified at the level of protein expression by gating onto the CD4+ fraction. Data are presented as the mean \pm SEM of 3 independent experiments (A) or are representative of 3 independent experiments (B).

of agonist anti-Tim-1 mAb, whereas Foxp3 expression remained high in the isotype control mAb Treg cultures (Figure 2B). Moreover, expression of Foxp3-triggered regulatory-associated factors, including CTL-associated antigen-4 (CTLA-4) and glucocorticoidinduced TNF receptor family-related protein (GITR) (25-28), was downregulated after provision of agonist anti-Tim-1 mAb (Figure $2 \mathrm{~B})$. In order to exclude the possibility that the increase in cell proliferation resulted from a stimulatory effect of anti-Tim-1 mAb upon a minor population of contaminating $\mathrm{CD}^{+}$effector cells, we repeated the MLR with $\mathrm{GFP}^{+}\left(\mathrm{Foxp}^{+}\right)$Tregs that were directly isolated from Foxp3-GFP knock-in mice (21) using a MoFlo cell sorter (purity >98\%). A serial analysis on day 2, 4, and 6 revealed that Foxp 3 gene expression increases markedly from day 2 through day 6 in the isotype control mAb cultures. In contrast, agonist antiTim-1 mAb in the MLR serves to powerfully blunt Foxp3 gene expression (Figure 2C). This decrease in Foxp3 gene expression was strengthened with time (from $34 \%$ decrease in gene expression on day 2 to $48 \%$ on day 6 in the agonist anti-Tim-1 mAb-supplemented culture vs. as isotype control $\mathrm{mAb}$-supplemented culture). The negative influence of anti-Tim-1 mAb on Foxp3 expression was confirmed at the protein level (Supplemental Figure 3).

IL-6 ${ }^{-/-}$mice bear a significantly elevated proportion of Foxp $3^{+}$ Tregs. Apparently, an absence of IL- 6 favors the generation or expansion of Tregs (29). Xu et al. have shown that provision of exogenous IL- 6 induced the differentiation of Foxp $3^{-} \mathrm{T}$ cells from $\mathrm{CD}^{+}{ }^{+} \mathrm{CD} 25^{+}$Foxp $^{+} \mathrm{T}$ cells (30). To determine whether the downregulation of Foxp 3 expression induced by agonist anti-Tim-1 $\mathrm{mAb}$ is IL-6 dependent, neutralizing anti-IL-6 mAb was added to the culture system (Figure 2D). With provision of neutralizing anti-IL-6 mAb, agonist anti-Tim-1 mAb did not alter the magnitude of Foxp3 gene expression.

The function of Tregs harvested from MLRs supplemented with 3B3 anti-Tim-1 mAb or IgG2a control cultures was then tested through their ability to inhibit the proliferative response of CFSElabeled Teffs to coated anti-CD3 mAb plus soluble anti-CD28 $\mathrm{mAb}$. Consistent with the decrease in Foxp3, GITR, CTLA-4, and IL-10 gene expression, the regulatory function of the anti-Tim-1stimulated Treg population was also markedly impaired (Figure $2 \mathrm{E})$. In comparison to the ability of proliferating Tregs from control MLRs to blunt $\mathrm{CD}^{+}$proliferation of Teffs to coated anti-CD3 $\mathrm{mAb}$ plus soluble anti-CD28 $\mathrm{mAb}$, the suppressive function of $3 \mathrm{~B} 3$ anti-Tim-1 mAb-treated alloreactive proliferating Tregs was vastly diminished (Figure 2D). Taken together, the transcriptional profile and functional data suggest that anti-Tim- $1 \mathrm{mAb}$ stimulation 


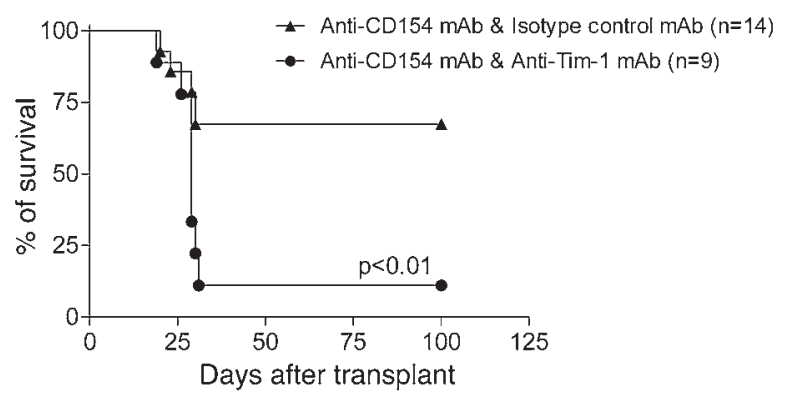

Figure 4

3B3 anti-Tim-1 mAb negates the tolerance-promoting effect of antiCD154 therapy in vivo. Diabetic C57BL/6 mice transplanted with DBA/2 pancreatic islet were treated with an anti-CD154 mAb in combination with either anti-Tim-1 mAb (circles; $n=9$ ), or the IgG2a isotype control mAb (triangles; $n=14$ ).

has the unique ability to deprogram Tregs and thereby weaken Treg immunoregulatory function.

Anti-Tim-1 mAb prevents the induction of $\mathrm{CD}^{+} \mathrm{Foxp}^{3^{+}}$Tregs from $C D 4^{+} \mathrm{Foxp}^{-} \mathrm{T}$ cells and promotes the differentiation of Th17 cells. The profound effect of anti-Tim-1 mAb on Foxp3 expression and regulatory functions in natural Tregs prompted us to examine whether anti-Tim- $1 \mathrm{mAb}$ would also prevent the induction of Foxp $3^{+}$Tregs from Foxp3- Teffs. Stimulation of $\mathrm{CD}^{+} \mathrm{GFP}(\mathrm{Foxp} 3)^{-} \mathrm{T}$ cells in the presence of isotype control $\mathrm{mAb}$ and TGF- $\beta$ induced robust expression of Foxp 3 transcripts (Figure $3 \mathrm{~A}$ ) and an increase in the frequency of Foxp $3^{+} \mathrm{CD} 4^{+} \mathrm{T}$ cells $(\approx 8 \%-10 \%)$ (Figure $\left.3 \mathrm{~B}\right)$. It is remarkable that conversion of such inducible Foxp $3^{+}$Tregs from naive $\mathrm{CD} 4^{+} \mathrm{GFP}(\mathrm{Foxp} 3)^{-} \mathrm{T}$ cells was completely blocked when anti-Tim-1 mAb was added to the culture (Figure 3, A and B).

Several groups including our own have reported an intriguing reciprocal relation between the development of Tregs and the development of pathogenic effector Th17 cells $(21,31,32)$. A common cytokine, TGF- $\beta$, is critical for the in vitro development of both Tregs and Th17 cells from $\mathrm{CD}^{+}{ }^{+}$Foxp $3^{-} \mathrm{T}$ cells. The differentiation into these subsets is determined by the presence or absence of inflammatory cytokines such as IL-6 (21), IL-1 $\alpha$ (32), and TNF- $\alpha$ (32). Interestingly, in line with the recent finding that IL-6 in conjunction with TGF- $\beta$ induces pathogenic Th17 cells while inhibiting the generation of inducible Foxp $3^{+}$Tregs (21) and with the observation that downregulation of Foxp3 expression induced by agonist anti-Tim-1 mAb is IL-6 dependent (Figure 2D), the combination of $3 \mathrm{~B} 3$ anti-Tim- $1 \mathrm{mAb}$ with TGF- $\beta$ promoted differentiation of Th17 cells as well (Figure $3 \mathrm{~B}$ ).

3B3 anti-Tim-1 mAb negates the tolerance-promoting effect of costimulation blockade in islet transplantation. Peripheral transplant tolerance can be regularly achieved through therapies that curtail the expansion of donor-destructive Teffs while preserving or strengthening donor-directed regulatory networks (33). Given the above data showing a detrimental effect of 3B3 anti-Tim-1 mAb on the balance between cytoprotective Tregs and effector cytopathic Th1/Th17 cells, we anticipated that in vivo administration of 3B3 anti-Tim-1 mAb would counteract the beneficial effects of tolerizing therapies in an allogeneic transplant model. To test this hypothesis, we chose an MHC-mismatched islet transplant model in which long-term survival of allogeneic islets can be achieved through administration of an anti-CD154 mAb (6, 34-36). Using this anti-CD154 mAb, tolerance results from inhibitory effects upon potency of donor-directed Teffs and enhances the suppressive function of donor-directed Tregs $(6,34)$. Indeed, in this model of MHC-incompatible islet transplantation, the ability of anti$\mathrm{CD} 154 \mathrm{mAb}$ treatment to prevent allograft rejection was largely negated by administration of anti-Tim- $1 \mathrm{mAb}$ (Figure 4; $P<0.01$; mean survival time, 29 days in the anti-Tim-1 mAb group vs. indefinite graft survival in the control group).

\section{Discussion}

In a study of the allograft response, we demonstrate that administration of 3B3 agonist anti-Tim-1 mAb prevents the induction of peripheral-type transplant tolerance by (a) enhancing the commitment and expansion of alloreactive T cells in the Th1/Th17 Teffs; (b) deprogramming natural Tregs at the molecular and functional levels; and (c) inhibiting the conversion of regulatory Foxp $3^{+}$cells from the naive Foxp3- T cells. In contrast, Ueno et al. show that treatment with an antagonist anti-Tim- $1 \mathrm{mAb}$ prolongs survival of heart allograft (37). The antagonist anti-Tim-1 mAb inhibits Teff responses and serves to enhance the potency of donor-reactive regulatory $\mathrm{CD} 4^{+} \mathrm{CD} 25^{+} \mathrm{T}$ cells. Opposing effects of the same agonist and antagonist anti-Tim-1 mAbs have been also noted in an EAE model (17) in which the agonist anti-Tim-1 mAb increases the severity of EAE, whereas the antagonist anti-Tim-1 mAb inhibits the development of EAE. Differences in the avidity of the binding to the IgV domain of the Tim-1 molecule by the agonist and antagonist anti-Tim-1 mAbs may determine the consequences of Tim-1 ligation. The agonist anti-Tim-1 mAb has avidity 17 -fold higher than that of the antagonist anti-Tim-1 mAb (17).

Wan and Flavell have generated a mouse in which endogenous Foxp3 gene expression is attenuated in Tregs (38). Using this strategy, they were able to compare the suppressive functions of Foxp $3^{\text {lo }} \mathrm{T}$ cells and Foxp $3^{\text {hi }} \mathrm{T}$ cells. They determined that reduced Foxp3 expression by natural Tregs results in diminished immunosuppressive functions (38). We now demonstrate that activation of CD $4^{+} \mathrm{Foxp}^{+} \mathrm{T}$ cells with an agonist anti-Tim- $1 \mathrm{mAb}$ leads to downregulation of Foxp3 expression, at both transcriptional and protein levels, as well as expression of Treg-associated molecules such as CD25, CTLA-4, and GITR (25-28). Activation of Tim-1 on $\mathrm{CD}^{+} \mathrm{Foxp}^{+} \mathrm{T}$ cells completely abrogates the in vitro suppressive functions of Foxp $3^{+} \mathrm{T}$ cells, as these anti-Tim- $1 \mathrm{mAb}$-stimulated Foxp $3^{\text {lo }} \mathrm{T}$ cells fail to regulate the proliferation of Teffs. Hence, ligation of Tim-1 serves to alter the commitment of Tregs from potent Foxp $3^{\text {hi }}$ to ineffective Foxp $3^{\text {lo }}$ Tregs.

A reciprocal relationship between commitment of naive $\mathrm{CD}^{+}$ $\mathrm{T}$ cells to the Th17 and Treg phenotypes has been discerned in several settings $(21,29)$. Depending on the presence or absence of certain proinflammatory cytokines such as IL- 6 or IL-21 in the milieu of antigen recognition, TGF- $\beta$ can support de novo differentiation of Th17 or Tregs $(21,32)$. We now show that engagement of the Tim-1 cell-surface receptor molecule upon T cells cultured with TGF- $\beta$ skews the commitment of naive T cells to Th 1 and Th17 and away from the Foxp $3^{\text {hi }}$ phenotype. Thus, our results lend further support to the concept of reciprocal commitment to Th17 and Treg phenotype, and we introduce a new means by which the commitment to the Teff and Treg phenotypes is regulated. While previous studies have centered upon the commitment of antigen-stimulated naive $T$ cells, we also demonstrate that the Foxp $3^{\text {hi }} \mathrm{T}$ cells are not terminally differentiated. Tim- 1 may serve to regulate the reciprocal developmental pathway for the genera- 
tion and maintenance of pathogenic and protective cells in the immune system. In the allograft response, Tim-1 ligation with an agonist anti-Tim-1 mAb promotes the commitment toward the Th17 and Th1 phenotypes and weakens commitment to the Foxp $3^{\text {hi }}$ phenotype. Of special importance is the fact that we have now identified a pathway that negatively regulates Foxp3 expression, even in committed Foxp $3^{\text {hi }} \mathrm{T}$ cells. It appears important to analyze the consequences, in physiological settings, of engagement of Tim- 1 by Tim- 4 , the Tim- 1 ligand that is expressed by selected antigen-presenting cell subsets (15). We hypothesize that interaction between Tim- $1^{+} \mathrm{T}$ cells and Tim- $4^{+}$DCs serves to heighten immune response by strengthening effector responses and downregulating the regulatory networks.

In conclusion, the $3 \mathrm{~B} 3$ agonist anti-Tim- $1 \mathrm{mAb}$ heightens cytopathic alloimmune responses and blocks the induction of transplant tolerance by: (a) favoring expansion and survival of Th1/ Th17 cells; (b) disarming Tregs at the molecular and functional levels; and (c) inhibiting the conversion of Teffs into Tregs. In contrast, treatment with an antagonist anti-Tim-1 mAb aids in the induction of transplant tolerance (37).

\section{Methods}

Mice. C57BL/6 (H-2 $/$ /CD45.2 $\left.2^{+}\right)$, B.6SJL-Ptprc ${ }^{\mathrm{a} P e p} 3^{\mathrm{b}} /$ BoyJ (H-2 $/$ CD $\left.45.1^{+}\right)$, and $\mathrm{DBA} / 2\left(\mathrm{H}-2^{\mathrm{d}}\right)$ mice were purchased from the Jackson Laboratory. The C57BL/6 Foxp3-GFP knock-in mice have been previously described (21). All mice were housed under standard conditions. All animal studies were approved by Institutional Review Board at Harvard Medical School.

$m A b s$ and reagents. All $\mathrm{mAbs}$ were purchased from eBioscience, except the anti-Tim-1 mAb (clone 3B3), which was produced in our laboratory.

Cell preparation. $\mathrm{CD} 4^{+} \mathrm{CD} 25^{-}$or $\mathrm{CD} 8^{+} \mathrm{CD} 25^{-} \mathrm{T}$ cells were obtained using negative selection. $\mathrm{CD} 4{ }^{+} \mathrm{CD} 25^{+} \mathrm{T}$ cells were obtained using the mouse $\mathrm{CD} 4{ }^{+} \mathrm{CD} 25^{+}$Regulatory $\mathrm{T}$ Cell Isolation Kit (Miltenyi Biotec). The purity of the T cell subset was greater than $95 \%$. CD $4{ }^{+} \mathrm{GFP}(\mathrm{Foxp} 3)^{+}$and CD4 ${ }^{+} \mathrm{GFP}($ Foxp3)- cells were sorted using a MoFlo high-speed cell sorter (Dako-Cytomation). Highly mature GM-CSF-derived DCs were obtained as previously described $(15,23)$.

In vitro MLR. C57BL $/ 6$ responder T cells were labeled with CFSE (Molecular Probes; Invitrogen) following the manufacturer's instructions and plated with stimulator cells in 96-well flat-bottom plates at a ratio of 4:1 in a final volume of $250 \mu \mathrm{l}$ of complete medium. Complete medium consisted of $1 \times$ MEM (Gibco/BRL; Invitrogen) supplemented with $2 \mathrm{mM}$ glutamine, $1 \mathrm{mM} \mathrm{Na}$ pyruvate, $0.1 \mathrm{mM}$ nonessential amino acids, $10 \% \mathrm{FBS}$, $0.3 \% \mathrm{Na}$ bicarbonate, $19 \mathrm{mM}$ HEPES, $50 \mu \mathrm{g} / \mathrm{ml}$ gentamicin, $5.5 \times 10^{-5} \mathrm{M}$ 2-mercaptoethanol, and $100 \mathrm{U} / \mathrm{ml}$ penicillin/streptomycin. IgG2a isotype control or anti-Tim-1 mAb was added to the culture medium at a final concentration of $2.5 \mu \mathrm{g} / \mathrm{ml}$. A CFSE profiling technique was used to assess the proliferation of the responder population by gating onto the $\mathrm{CD}^{+}$ population. Cells were counterstained with annexin V-PE (BD Biosciences - Pharmingen) in order to determine the extent of cell death.

T cell suppression assay. CFSE-labeled C57BL/6 (CD 45.2 $\left.{ }^{+}\right) \mathrm{CD}^{+} \mathrm{CD} 25^{+} \mathrm{T}$ cells were cultured with mature DBA/2 DCs at a ratio of 4:1. After 6 days of culture, proliferating $\mathrm{CD} 4{ }^{+} \mathrm{CD} 25^{+} \mathrm{T}$ cells $\left(\mathrm{CFSE}^{\mathrm{dim}}\right)$, isolated by the MoFlo high-speed cell sorter, were mixed with $\mathrm{CD} 45.1^{+} \mathrm{CD} 4^{+} \mathrm{CD} 25^{-} \mathrm{T}$ cells at vari- ous ratios and placed into anti-CD3-coated plates in the presence of 2.5 $\mu \mathrm{g} / \mathrm{ml}$ soluble anti-CD28 for 4 days. CFSE profiles of the $\mathrm{CD} 3{ }^{+} \mathrm{CD} 45.1^{+}$ Teffs were analyzed by flow cytometry.

Induction of Foxp $3^{+}$Tregs from Teffs in vitro. Purified C57BL $/ 6 \mathrm{CD} 4^{+} \mathrm{GFP}$ (Foxp3)- Teffs were stimulated with mature syngeneic DCs at a ratio of 4:1 plus anti-CD3 mAb $(5 \mu \mathrm{g} / \mathrm{ml})$ with IgG2a mAb or anti-Tim-1 mAb $(5 \mu \mathrm{g} / \mathrm{ml})$ and in the presence or absence of TGF- $\beta(1 \mathrm{ng} / \mathrm{ml}$; BioSource; Invitrogen) for 3 days. After intracellular Foxp3 staining, induction of $\mathrm{Foxp}^{+}$cells from Teffs was determined by flow cytometry by gating on the $\mathrm{CD} 4{ }^{+} \mathrm{Foxp} 3^{+}$cells and presented as percentage of total $\mathrm{CD}^{+}$cells.

Intracellular cytokine analysis. Before intracellular staining, cells were restimulated for 4 hours with $50 \mathrm{ng} / \mathrm{ml} \mathrm{PMA} \mathrm{and} 400 \mathrm{ng} / \mathrm{ml}$ ionomycin in the presence of $1 \mu \mathrm{g} / \mathrm{ml} \mathrm{GolgiPlug}$ (BD Biosciences). Intracellular staining was conducted using the intracellular staining kit (eBioscience) according to the manufacturer's protocols.

Real-time quantitative PCR. The expression of genes of interest was analyzed using TaqMan primer-probe sets purchased as Assay-on-Demand from Applied Biosystems and normalized to the expression of GAPDH. The list of TaqMan primer-probe sets used is presented in Supplemental Table 1 . Transcript levels were calculated according to the $2^{-\Delta \Delta \mathrm{Ct}}$ method as described by the manufacturer and expressed in arbitrary units. Histograms represent the mean of the gene expression detected in a set of samples and error bars represent SEM.

Islet transplantation. DBA/2 (H-2 $)$ into C57BL/6 $\left(\mathrm{H}-2^{\mathrm{b}}\right)$ islet transplantation was performed as previously described (39). The tolerance-promoting protocol consisted of $250 \mu \mathrm{g}$ hamster anti-mouse CD154 (MR1, IgG2a, ATCC HB11048) administered i.p. on days 0 and 2 after transplantation. MR1 anti-CD154 mAb treatment was coadministered with 2 doses $(250 \mu \mathrm{g}$ each) of either rat IgG2a (isotype control) or 3B3 anti-Tim-1 mAb.

Statistics. Mann-Whitney $U$ tests were performed to compare gene-specific transcript levels present in cells harvested from control (IgG2a) and anti-Tim-1 mAb-treated MLRs. Islet allograft survival was analyzed by the Kaplan-Meier test. $P$ values of less than 0.05 were considered significant.

\section{Acknowledgments}

This work was supported by grants from the Société de Néphrologie (to C. Mariat), the Société Francophone de Transplantation (to C. Mariat), the National Institute of Allergy and Infectious Disease (to X.X. Zheng, V. Kuchroo, D. Umetsu, and T.B. Strom), and the Juvenile Diabetes Research Foundation Center for Immunologic Tolerance at Harvard Medical School (to T.B. Strom).

Received for publication May 2, 2007, and accepted in revised form November 13, 2007.

Address correspondence to: Terry B. Strom, Transplant Research Center, Beth Israel Deaconess Medical Center, H.I.M.-1, Room 1026, 77 Avenue Louis Pasteur, Boston, Massachusetts 02115, USA. Phone: (617) 667-0850; Fax: (617) 667-0923; E-mail: tstrom@ bidmc.harvard.edu.

Nicolas Degauque and Christophe Mariat are co-first authors and contributed equally to this work.

\footnotetext{
1. Kuchroo, V.K., Umetsu, D.T., DeKruyff, R.H., and Freeman, G.J. 2003. The TIM gene family: emerging roles in immunity and disease. Nat. Rev. Immunol. 3:454-462.

2. Chae, S.C., Park, Y.R., Lee, Y.C., Lee, J.H., and Chung, H.T. 2004. The association of TIM-3 gene polymorphism with atopic disease in Korean population. Hum. Immunol. 65:1427-1431.
}

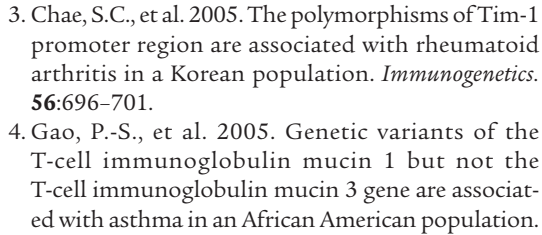

3. Chae, S.C., et al. 2005. The polymorphisms of Tim-1 promoter region are associated with rheumatoid arthritis in a Korean population. Immunogenetics. 56:696-701.

4. Gao, P.-S., et al. 2005. Genetic variants of the T-cell immunoglobulin mucin 1 but not the T-cell immunoglobulin mucin 3 gene are associated with asthma in an African American population.

J. Allergy Clin.Immunol. 115:982-988.

5. Sabatos, C.A., et al. 2003. Interaction of Tim-3 and Tim-3 ligand regulates $T$ helper type 1 responses and induction of peripheral tolerance. Nat. Immunol. 4:1102-1110.

6. Sanchez-Fueyo, A., et al. 2003. Tim-3 inhibits T helper type 1-mediated auto- and alloimmune responses and promotes immunological tolerance. 
Nat. Immunol. 4:1093-1101.

7. Chakravarti, S., et al. 2005. Tim-2 regulates T helper type 2 responses and autoimmunity. J. Exp. Med. 202:437-444.

8. Zhu, C., et al. 2005. The Tim-3 ligand galectin-9 negatively regulates $\mathrm{T}$ helper type 1 immunity. Nat. Immunol. 6:1245-1252.

9. Meyers, J.H., Sabatos, C.A., Chakravarti, S., and Kuchroo, V.K. 2005. The TIM gene family regulates autoimmune and allergic diseases. Trends Mol. Med. 11:362-369.

10. Mariat, C., et al. 2005. Regulation of T cell dependent immune responses by TIM family members. Philos. Trans. R Soc. Lond. B Biol. Sci. 360:1681-1685.

11. McIntire, J.J., Umetsu, D.T., and DeKruyff, R.H. 2004. TIM-1, a novel allergy and asthma susceptibility gene. Springer Semin. Immunopathol. 25:335-348.

12. Umetsu, D.T., McIntire, J.J., and DeKruyff, R.H. 2005. TIM-1, hepatitis A virus and the hygiene theory of atopy: association of TIM-1 with atopy. J. Pediatr. Gastroenterol. Nutr. 40(Suppl. 1):S43.

13. Umetsu, S.E., et al. 2005. TIM-1 induces T cell activation and inhibits the development of peripheral tolerance. Nat. Immunol. 6:447-454.

14. Khademi, M., et al. 2004. T Cell Ig- and mucindomain-containing molecule-3 (TIM-3) and TIM-1 molecules are differentially expressed on human Th1 and Th2 cells and in cerebrospinal fluidderived mononuclear cells in multiple sclerosis. J. Immunol. 172:7169-7176.

15. Meyers, J.H., et al. 2005. TIM-4 is the ligand for TIM-1, and the TIM-1-TIM-4 interaction regulates $\mathrm{T}$ cell proliferation. Nat. Immunol. 6:455-464.

16. de Souza, A.J., Oriss, T.B., O’Malley, K.J., Ray, A., and Kane, L.P. 2005. T cell Ig and mucin 1 (TIM-1) is expressed on in vivo-activated $\mathrm{T}$ cells and provides a costimulatory signal for $\mathrm{T}$ cell activation. Proc. Natl. Acad. Sci. U. S. A. 102:17113-17118.

17. Xiao, S., et al. 2007. Differential engagement of Tim-1 during activation can positively or negatively costimulate $T$ cell expansion and effector function. J. Exp. Med. 204:1691-1702.
18. Wells, A.D., Gudmundsdottir, H., and Turka, L.A. 1997. Following the fate of individual t cells throughout activation and clonal expansion. Signals from $\mathrm{T}$ Cell receptor and CD28 differentially regulate the induction and duration of a proliferative response. J. Clin. Invest. 100:3173-3183.

19. Li, X.C., et al. 2001. IL-15 and IL-2: a matter of life and death for T cells in vivo. Nat. Med. 7:114-118.

20. Petschner, F., et al. 1998. Constitutive expression of $\mathrm{Bcl}-\mathrm{xL}$ or $\mathrm{Bcl}-2$ prevents peptide antigeninduced $\mathrm{T}$ cell deletion but does not influence $\mathrm{T}$ cell homeostasis after a viral infection. Eur. J. Immunol. 28:560-569.

21. Bettelli, E., et al. 2006. Reciprocal developmental pathways for the generation of pathogenic effector TH17 and regulatory T cells. Nature. 441:235-238.

22. Yamazaki, S., et al. 2003. Direct expansion of functional CD25+ CD4+ regulatory $\mathrm{T}$ cells by antigenprocessing dendritic cells. J. Exp. Med. 198:235-247.

23. Sanchez-Fueyo, A., et al. 2006. Specificity of $\mathrm{CD} 4+\mathrm{CD} 25+$ regulatory $\mathrm{T}$ cell function in alloimmunity. J. Immunol. 176:329-334.

24. Yamazaki, S., et al. 2006. Effective expansion of alloantigen-specific Foxp3+ CD25+ CD4+ regulatory $\mathrm{T}$ cells by dendritic cells during the mixed leukocyte reaction. Proc. Natl. Acad. Sci. U. S. A. 103:2758-2763.

25. Fontenot, J.D., Gavin, M.A., and Rudensky, A.Y. 2003. Foxp3 programs the development and function of CD4+CD25+ regulatory T cells. Nat. Immunol. 4:330-336.

26. Hori, S., Nomura, T., and Sakaguchi, S. 2003. Control of regulatory $\mathrm{T}$ cell development by the transcription factor Foxp3. Science. 299:1057-1061.

27. Fontenot, J.D., Rasmussen, J.P., Gavin, M.A., and Rudensky, A.Y. 2005. A function for interleukin 2 in Foxp3-expressing regulatory T cells. Nat. Immunol. 6:1142-1151.

28. Khattri, R., Cox, T., Yasayko, S.A., and Ramsdell, F. 2003. An essential role for Scurfin in CD4+CD25+ $\mathrm{T}$ regulatory cells. Nat. Immunol. 4:337-342.

29. Korn, T., et al. 2007. IL-21 initiates an alternative pathway to induce proinflammatory $\mathrm{T}(\mathrm{H}) 17$ cells. Nature. 448:484-487.

30. Xu, L., Kitani, A., Fuss, I., and Strober, W. 2007. Cutting edge: regulatory $\mathrm{T}$ cells induce $\mathrm{CD} 4+\mathrm{CD} 25$ Foxp3- $\mathrm{T}$ cells or are self-induced to become Th17 cells in the absence of exogenous TGF-beta. J. Immunol. 178:6725-6729.

31. Mangan, P.R., et al. 2006. Transforming growth factor-beta induces development of the T(H)17 lineage. Nature. 441:231-234.

32. Veldhoen, M., Hocking, R.J., Atkins, C.J., Locksley, R.M., and Stockinger, B. 2006. TGFbeta in the context of an inflammatory cytokine milieu supports de novo differentiation of IL-17-producing T cells. Immunity. 24:179-189.

33. Zheng, X.X., Sanchez-Fueyo, A., Domenig, C., and Strom, T.B. 2003. The balance of deletion and regulation in allograft tolerance. Immunol. Rev. 196:75-84

34. Jarvinen, L.Z., Blazar, B.R., Adeyi, O.A., Strom, T.B., and Noelle, R.J. 2003. CD154 on the surface of $\mathrm{CD} 4+\mathrm{CD} 25+$ regulatory $\mathrm{T}$ cells contributes to skin transplant tolerance. Transplantation. 76:1375-1379.

35. Quezada, S.A., et al. 2005. Analysis of the underlying cellular mechanisms of anti-CD154-induced graft tolerance: the interplay of clonal anergy and immune regulation. J. Immunol. 175:771-779.

36. Parker, D.C., et al. 1995. Survival of mouse pancreatic islet allografts in recipients treated with allogeneic small lymphocytes and antibody to CD40 ligand. Proc. Natl. Acad. Sci. U. S. A. 92:9560-9564.

37. Ueno, T., et al. 2008. The emerging role of T cell immunoglobulin mucin 1 in alloimmune responses in an experimental mouse transplant model. doi:10.1172/JCI32451.

38. Wan, Y.Y., and Flavell, R.A. 2007. Regulatory T-cell functions are subverted and converted owing to attenuated Foxp3 expression. Nature. 445:766-770.

39. Sanchez-Fueyo, A., Weber, M., Domenig, C., Strom, T.B., and Zheng, X.X. 2002. Tracking the immunoregulatory mechanisms active during allograft tolerance. J. Immunol. 168:2274-2281. 